\title{
Psicoterapia on-line: uma revisão de literatura
}

\author{
Online psychotherapy: a literature review
}

\author{
Henrique Borba Bittencourt', Caroline Cassal Rodrigues², Gabriel Licoski dos \\ Santos', Jaqueline Batista da Silva ${ }^{4}$, Laura Gonçalves de Quadros ${ }^{5}$, Lucas Santos \\ Mallmann ${ }^{6}$, Pâmela Soares Bratkowski' e Renata Isabel Fedrizzi ${ }^{8}$
}

\begin{abstract}
Resumo: $O$ presente artigo teve por objetivo realizar uma revisão narrativa da literatura a fim de compreender os conceitos envoltos no processo terapêutico on-line. Tal pesquisa se mostra importante devido ao grande destaque que a temática ganhou nos tempos atuais em decorrência da crise provocada pela Covid-19, tendo em vista os efeitos na saúde mental e na forma de fazer psicologia. Os resultados encontrados apontam para a semelhança da efetividade do tratamento on-line quando comparado ao modelo clássico, a existência de peculiaridades do setting terapêutico, suas vantagens e ainda, sua importância tendo em vista as mudanças nas formas de se relacionar. Além disso, é destacada a relativa escassez de trabalhos sobre a temática, sendo importante o incentivo à produção destes.
\end{abstract}

Palavras-chave: Psicoterapia On-line; Terapia On-line; Atendimentos psicológicos pela internet.

\begin{abstract}
This article aims at conducting a literature narrative review in order to understand the concepts involved in the online therapeutic process. This research is important because of the great prominence that this subject has gained in the current times as a result of the crisis caused by Covid-19, given the effects on mental health and the way of doing psychology. The results found point to the similarity of the effectiveness of online treatment when compared to the classic model, the existence of peculiarities of the therapeutic setting, its advantages and also its importance given the changes in the forms of relationship. In addition to the lack of works on this subject, it's important to encourage their development.
\end{abstract}

Keywords: Online Psychotherapy; E-Therapy; Psychological care over the internet.

\footnotetext{
1 Estudante de Psicologia no Centro Universitário Cenecista de Osório. Coordenador da comissão de pesquisa da Liga de Estudantes e RecémFormados da SPRGS (L.E.R.). E-mail: iqueb2@hotmail.com .

2 Estudante de Psicologia na Faculdade Cesuca, Coordenadora da comissão de comunicação da Liga de Estudantes e RecémFormados da SPRGS (L.E.R.). E-mail: carol.cassalrodrigues@gmail.com .

3 Estudante de Psicologia no Centro Universitário Cenecista de Osório. Ligante da comissão de comunicação da Liga de Estudantes e RecémFormados da SPRGS (L.E.R.). E-mail: biellicoski@gmail.com .

${ }^{4}$ Estudante de Psicologia na Faculdade Anhanguera. Coordenadora da comissão administrativa da Liga de Estudantes e RecémFormados da SPRGS (L.E.R.). E-mail: jackbatistsilva@gmail.com .

${ }^{5}$ Estudante de Psicologia na Faculdade Anhanguera. Coordenadora da comissão de extensão da Liga de Estudantes e RecémFormados da SPRGS (L.E.R.). E-mail: lauraexecute@gmail.com .

${ }^{6}$ Estudante de Psicologia na Faculdade Anhanguera. Ligante da comissão de comunicação da Liga de Estudantes e RecémFormados da SPRGS (L.E.R.). E-mail: lucassantosmallmann@gmail.com .

7 Psicóloga formada pela UNISINOS. Psicanalista em formação pela Sigmund Freud Associação Psicanalítica (Sig), Membro efetivo e diretora científica da SPRGS. E-mail: pamelasoaresb@gmail.com .

8 Psicóloga formada pela PUCRS. Psicanalista em formação pela Sig. Membro efetivo da SPRGS. E-mail: renataisabelfedrizzi@ gmail.com .
} 


\section{Introdução}

0 presente artigo foi proposto a partir da realização do evento "Atendimento on-line e exercício da psicologia: um diálogo necessário sobre novas resoluções", organizado pela Liga de Estudantes e Recém-formados (L.E.R.). As discussões do evento suscitaram questionamentos e investigações sobre o tema pelos membros da L.E.R., que buscaram respostas para tais indagações por meio de pesquisas.

Enquanto se realizavam os primeiros estudos sobre 0 tema por parte dos autores, a pandemia do coronavírus eclodiu no mundo, dando ainda mais relevância à temática proposta, visto as novas questões e inquietações sobre atendimentos psicológicos on-line. Além disso, o formato deste trabalho precisou ser repensado frente à necessidade de abarcar as mudanças que estavam ocorrendo rapidamente para os psicólogos.

0 ano de 2020 pode ser considerado um marco para a psicologia no que diz respeito aos atendimentos on-line. Isto porque, até então, os profissionais ainda estavam se apropriando da Resolução CFP no 11/2018 do Conselho Federal de Psicologia (2018), que visa a ampliação das possibilidades de serviços oferecidos por meio de tecnologias de informação e comunicação (TICS), e viram-se diante da necessidade de trabalhar preferencialmente desta forma devido à pandemia de Covid-19.

Os psicólogos, assim como o restante da população, foram surpreendidos pelo novo coronavírus, que teve seus primeiros casos confirmados no final de 2019 na China (Organização Pan-Americana de Saúde \& Organização Mundial da Saúde 2020). Em pouco tempo, a doença se disseminou pelo mundo, evoluindo de uma epidemia para uma pandemia, instaurando um panorama de emergência de saúde pública internacional (Organização Pan-Americana de Saúde \& Organização Mundial da Saúde 2020) e mudando a rotina de milhões de pessoas nos esforç̧os para o seu controle. 0 primeiro caso registrado em terras brasileiras ocorreu no dia 26 de fevereiro de 2020, em São Paulo, e passados 99 dias foram registrados cerca de 555.383 casos confirmados e 31.199 óbitos por infecção pelo vírus (Organização Pan-Americana de Saúde \& Organização Mundial da Saúde, 2020).

Devido à rápida propagação do vírus, as pessoas foram orientadas a manter o distanciamento social, e apenas os serviços considerados essenciais à população continuaram abertos. Em consonância com essa situação, o CFP orientou a categoria e publicou a Resolução CFP no 04/2020, flexibilizando alguns dispositivos da Resolução (FP no 11/2018 com o objetivo de manter 0 atendimento à população em isolamento (Conselho Federal de Psicologia, 2020a). A partir dessa regulamentação, os psicólogos puderam manter os serviços prestados por meio das TICS desde que fizessem seu cadastro na plataforma e-Psi e observassem as exigências do Código de Ética Profissional estabelecido pela Resolução CFP no 10/2005 (Conselho Federal de Psicologia, 2005a).

Segundo Fabiane Konowaluk (como citado por Conselho Federal de Psicologia, (2020b), tesoureira do Conselho Regional de Psicologia do Rio Grande do Sul (CRPRS), o serviço de cadastro para atendimento on-line chegou ao registro de mais de 30 mil acessos em um dia, provocando um colapso na plataforma de cadastro. Diante desse cenário, o CFP autorizou que os psicólogos atuassem antes mesmo de receberem o parecer do respectivo Conselho Regional de Psicologia (CRP) (Conselho Federal de Psicologia, 2020b).

Embora os atendimentos psicológicos on-line ainda sejam uma prática recente, diante do momento em que a sociedade se encontra, novas formas de atuação do psicólogo nesse âmbito se fazem necessárias, sendo cada vez mais objeto de estudo e de novas orientações para que o exercício da profissão prossiga de forma ética. Por este motivo, os membros da L.E.R. decidiram escrever este artigo e contribuir com reflexões e informações sobre esta modalidade de atendimento.

\section{Método}

A metodologia utilizada para 0 artigo foi 0 de revisão narrativa de literatura, visto que este é um método que permite ao autor realizar a análise e interpretação de um tema de forma ampla sob o ponto de vista teórico e contextual (Rother, 2007, Elias et al., 2012, Brizola e Fantin, 2016). Este método de estudo, segundo Rother (2007), baseia-se na análise da literatura publicada em livros e artigos de revistas.

Para a realização deste estudo foram utilizados autores que se enquadram no tema proposto, além da realização de buscas nas bases de dados Lilacs, Pepsic, Scielo e Pubmed, com as palavras chaves"psicoterapia on-line (online psychotherapy)"e "terapia on-line (online therapy)" ou "atendimento psicológico on-line". Com base nesta busca, foi possível identificar que ainda há poucos estudos sobre o tema no âmbito nacional, visto que, depois de utilizados como critérios a exclusão de artigos com mais de cinco anos e que não dissertem sobre atendimento psicológico on-line, bem como dos repetidos, chegou-se a apenas 52 artigos, o que fomentou o desejo de produzir um estudo sobre 0 tema.

Devido ao escasso material encontrado em língua portuguesa, utilizou-se também para construção desta pesquisa, informações presentes em livros, resoluções e materiais de divulgação do CFP. Além disto, também foi utilizada literatura complementar indicada por meio das leituras dos artigos. Cabe ressaltar que, depois das buscas, foram utilizados basicamente autores que apresentassem considerações consistentes para o presente estudo e que discorressem sobre os tópicos escolhidos.

\section{Resultados e discussão}

Os resultados e discussão apresentados a seguir foram divididos em tópicos conforme os objetivos deste artigo, são eles: pós-modernidade de psicoterapia, presença física e virtual, atendimento on-line e a história, setting e contrato síncrono e assíncrono.

\section{Pós-modernidade e psicoterapia}

A pós-modernidade teve seu advento no final do século XIX e início do século XX, com o impacto do desenvolvimento tecnológico sobre o saber, 0 intenso processo de globalização e a instauração do cenário cibernético (Dockhorn \& Macedo, 2008). Com essas mudanças, a interação social avançou progressivamente, passando pela tipografia e o telégrafo (Neto, 2020) e, atualmente, sendo conduzida por meio de uma rede de informações vasta e dinâmica, a internet (Witt \& Rostirola, 2020). Dessa maneira, um evento que ocorre, por exemplo, na China, em poucos segundos vira notícia no mundo e afeta direta ou indiretamente a todos.

Segundo Neto (2020), a extinção das antigas barreiras geográficas e o fato de um mesmo evento afetar todos os indivíduos do mundo como se fosse 
uma vizinhança remete à ideia de "aldeia global", desenvolvida por Marshall McLuhan. Ainda de acordo com Neto (2020), essa fluída dinâmica global e de interconexões das esferas econômicas, sociais e culturais, principalmente mediadas pelas TICs, diz respeito ao processo globalização.

Nessa linearidade de pensamento, Ceroni (2017) refere que as TICS encurtam as distâncias e otimizam o tempo, inclusive no setting terapêutico, proporcionando uma atuação do psicólogo para além do espaço físico. Pensando nisso, o uso das tecnologias no atual cenário mundial proporciona aos psicólogos a possibilidade de repensarem sobre suas atuações, visto que a internet se tornou uma importante ferramenta para conectar as pessoas.

Dessa forma, cabe aos Conselhos de Psicologia e aos psicólogos adaptarem suas interações enquanto atores sociais diante de um número cada vez mais significativo de pessoas que se comunicam virtualmente e até mesmo participam de atividades que ocorrem no espaço virtual. Torna-se necessário rever como a escuta do sujeito globalizado, alinhada com um olhar para 0 corpo tecnológico proposto por Ceroni (2017), constituiu-se e ainda vem se desenvolvendo em meio aos desafios presentes no território on-line.

Ainda segundo a autora, o sujeito contemporâneo se encontra conectado quase que integralmente ao meio virtual e, desta forma, consegue se fazer presente mesmo a longas distâncias. Isto porque, o que define a qualidade das relações é a capacidade que o sujeito tem para desenvolver seus vínculos com os outros e adaptar-se, o que está para além dos limitadores de um sinal de Wi-Fi ou de um acesso ao digital (Ceroni, 2017).

\section{Presença física e virtual}

A partir do avanço das tecnologias, que permitiram ao humano interagir tanto física quanto virtualmente, muitos conceitos foram se tornando obsoletos, ao passo que foram sendo desenvolvidas e atualizadas as noções de escrita, identidade e distância. Dentre essas mudanças, pode-se pensar que 0 conceito de corpo também adquiriu um novo olhar no mundo contemporâneo, visto as transformações na interação e diferenças entre as formas de se estar presente (Rosa, Santos \& Faleiros, 2016). 0 corpo dessa nova sociedade que se apresenta, chamada de rede, é mais complexo, constituído por meio de um compilado de dados pessoais que não se opõe ao real, porém, interliga as sensações do ciberespaço e facilita a comunicação e experimentação dos outros "eus" e outras maneiras de ser no mundo. A partir disso, dá-se lugar a outras representações de emoção e descobertas deste "eu" (Baldanza, 2006).

Os significados atribuídos ao virtual e ao real são amplamente discutidos por Pierre Lévy (1996) em seu livro "0 que é virtual". Segundo o autor, os campos que compreendem o real e o virtual apresentam potenciais diferentes, mas ambos possuem a capacidade de se atualizarem através do tempo. Lévy também refere que a palavra real vem do latim medieval do realis, que procede de res (coisa), pertencente à classificação do senso comum, do indivíduo referente à presença, portanto, para ele, existente de fato, tal como o computador, a cadeira e a casa.

De acordo com o Dicionário Aurélio da Língua Portuguesa (Ferreira, 1999), virtual vem do latim virtus, que significa força e potência, aquilo que existe como elemento que se desloca entre o potencial e o objetivo da transmissão de informação, necessitando de interconexão para atualizar-se, inovando o conceito de estar presente, não se opondo ao real, mas divergindo do atual. Em virtude das novas configurações das relações através de TICS, nas quais o comparecimento físico deixa de ser critério para estabelecer a existência, a cultura é transformada, e restabelecem-se novas formas de interação, que não estão diretamente ligadas ao conceito de tempo, mas de transmissão de comunicação (Rosa, Santos \& Faleiros, 2016).

A unidade tempo, em seu processo de virtualização, dispensa a necessidade de lugar e proporciona novos modos de criação, sendo esse processo chamado de cibercultura. 0 modelo tridimensional de virtualidade de Pierre Lévy (1996) destaca a relação entre o senso comum, o filosófico e o tecnológico como complementares para a realidade presente em cada ato. Para Galvão (2016), a parte mais significante da obra de Lévy consiste nas simulações da realidade externa. Nesta imersão virtual, o indivíduo tem total controle deste ambiente, o que para muitos tratamentos psicológicos têm-se mostrado eficazes. Um exemplo é com relação aos casos de fobia social, em que, nestes tratamentos, o psicólogo controla a intensidade da exposição ao ambiente ansiogênico, a fim de dessensibilizar o paciente por meio desta prática.

\section{Atendimento on-line e a história}

Em uma gama de países como Austrália, Estados Unidos, China e Reino Unido o uso das tecnologias de informação e comunicação em psicoterapia já é uma prática amplamente aceita e difundida. Contudo, mesmo nestes países, seu início foi marcado por receios e questionamentos. Inicialmente, acreditava-se que esta prática poderia levar a um empobrecimento na relação terapêutica, dificuldades a respeito da confidencialidade das relações, problemas relacionados a questões legais e ao treino de psicólogos versados em tal modalidade. Apesar desse estranhamento inicial, com a difusão do alcance das TICs, emergiu a necessidade da elaboração de estudos a respeito desta forma de fazer psicoterapia (Rodrigues \& Tavares, 2016).

Nesse contexto, foram desenvolvidas, principalmente em âmbito internacional, diversas pesquisas sobre a eficácia da psicoterapia on-line. Surgiram, assim, uma série de estudos que comprovam que os efeitos desta modalidade são muito similares aos al cançados nos atendimentos realizados em consultórios, seja na redução de sintomas ou na formação do vínculo terapêutico. Tais conclusões são baseadas em estudos sobre o tratamento de pacientes com Transtornos Depressivos (Josephine, Josefine, Phillip, David \& Harald, 2017), Transtorno do Estresse Pós-Traumático (Simblett, Birch, Matcham, Yaguez \& Morris, 2017), Transtornos Alimentares (Loucas et al., 2014), Transtorno de Ansiedade Generalizada (Richards, Richardson, Timulak \& McElvaney, 2015) e ainda pesquisas a respeito da efetividade geral do atendimento on-line (Barak, Hen, Boniel-Nissim \& Shapira, 2008).

Além disso, esta modalidade apresenta algumas vantagens em relação ao modelo tradicional de psicoterapia. Alguns exemplos são a ampliação da rede de acesso ao serviço, a diminuição dos custos com espaço e maior receptividade de alguns pacientes. (Proudfoot et al., 2011). 0 anonimato, por exemplo, pode vir a auxiliar no atendimento com pacientes introvertidos, com transtorno de ansiedade e ainda com disforia da imagem corporal. Nesse sentido, o uso de técnicas de psicoterapia on-line tem se mostrado efetivo e até mais adequado para determinados transtornos e contextos (Rodrigues \& Tavares, 2016).

No entanto, no âmbito nacional, tal prática ainda não se encontra tão difundida em termos de pesquisa e disponibilidade. Podemos destacar na construção histórica das normativas desta temática as resoluções do CFP entre os anos de 2000 a 2018. É importante ressaltar que estas são de caráter 
substitutivo e não cumulativo, ou seja, uma revogou a outra no ato de sua implantação.

As Resoluções do CFP nº 003/2000, 012/2005 e 011/2012 permitiam 0 atendimento psicoterapêutico apenas em caráter experimental tendo em vista a escassez de produções, sobre a quantidade de pesquisas nacionais que comprovam sua efetividade, em suas determinadas épocas. (Conselho Federal de Psicologia, 2000, 2005b, 2012). Com essa necessidade em mente, pesquisas foram desenvolvidas ao longo do tempo com o foco em tal finalidade. Como exemplo, tem-se o estudo de Pieta (2014), em que foram realizados atendimentos on-line síncronos com vinte e quatro pacientes randomizados, encontrando-se resultados favoráveis quanto à formação da relação terapêutica e a efetividade do tratamento on-line.

Nesse contexto, a Resolução no 11/2018 (Conselho Federal de Psicologia, 2018) trouxe algumas alterações, dentre as quais cabe destacar: o número de sessões máxima não mais se encontra delimitado; 0 atendimento psicoterapêutico agora é permitido - visto que previamente só ocorria em caráter experimental -; a exigência de cadastro de um site passou a ser do próprio indivíduo; 0 atendimento de crianças e adolescentes pode ocorrer mediante consentimento expresso de ao menos um dos seus responsáveis legais; e fica vedado 0 atendimento à pessoa em situação de violação de direito ou violência, devendo esse tipo de serviço ser prestado presencialmente.

Devido à pandemia do coronavírus, além de os psicólogos poderem iniciar os atendimentos on-line antes de receberem a aprovação dos Conselhos Regionais de Psicologia devido à suspensão dos Artigos 3 e 4 da Resolução CFP no 11/2018, também foram suspensos os Artigos 6, 7 e 8 com a Resolução no 04/2020. Sendo assim, ficam permitidos os atendimentos a pessoas e grupos em situações de risco, emergência, desastre e violação de direito até que sobrevenha uma nova Resolução (Conselho Federal de Psicologia, 2020a).

\section{Setting e contrato}

Pensando na prática terapêutica, o setting é um espaço que se apresenta para propiciar a estruturação simbólica de um tratamento e é nele que se integram as condições técnicas básicas para a intervenção do psicólogo (Barros, 2013). Green (2008) define este termo proposto por Winnicott como o conjunto de elementos que compõem um ambiente estruturante. Posto isto, faz parte da definição de setting a fixação do contrato de trabalho, incluindo pagamentos, horários e combinações que nortearão a relação entre psicólogo e paciente.

0 contrato, que faz parte dessa função estruturante na formação do setting, cria um alicerce, que permite observar as manifestações do inconsciente, resistências e reações de transferência e contratransferência (Lucion \& Knijnik, 2015). Algumas noções básicas de contrato foram apresentadas por Freud em dois de seus escritos: Recomendações aos médicos que exercem a psicanálise (Freud, 1912-1996) e Sobre o início do tratamento (1913-1996), nos quais ele ressalta pontos importantes sobre a constituição deste. Freud (1913-1996) compara o tratamento com o jogo de xadrez, no qual as aberturas e os finais são limitadas às regras, mas no percurso do jogo é permitido uma infinita variedade de jogadas que desafiam qualquer descrição deste tipo. Ele ainda descreve que o que assegura o tratamento não é apenas o que envolve o setting, mas, sim, a posição simbólica ocupada pelo psicólogo.

Na modalidade on-line, o setting também se faz presente, mas com algumas particularidades específicas. 0 atendimento passa a ocorrer por intermédio de dispositivos tecnológicos, como computadores e aparelhos telefônicos. Dessa forma, é através da viabilidade do espaço de escuta que se torna possível preservar esse espaço. Fink (2007/2017) reflete sobre os fenômenos imaginários possíveis nesta prática realizada por meio do telefone. 0 paciente tem liberdade para identificar o psicólogo com suas próprias imagens visuais, como uma tela de projeção em branco, baseadas na forma de falar, tom da voz e entonação. Se para o autor é pela fala que ocorre um tratamento, é muito importante que psicólogo e paciente sejam capazes de ouvir um ao outro. Posto isso, mesmo nas sessões que ocorrem por algum dispositivo, é possível seguir ocorrendo a interpretação das associações do paciente.

Nóbrega (2015) afirma que, se forem seguidos princípios teóricos e técnicos básicos, como a proposta de um setting consistente e ético, torna-se viável pensar em uma flexibilidade da definição de processo psicoterapêutico. A constituição de um tratamento é atravessada por um ambiente em rápida evolução, no qual novas tecnologias e a cultura devem ser consideradas.

Ao encontro dessas ideias, Silva, Siegmund e Brendmeier (2015) apontam que o tratamento on-line está ganhando espaço para possibilitar um novo formato de acesso aos pacientes, mais sintonizado com a contemporaneidade e não para desconsiderar o trabalho presencial do psicólogo. 0 atual momento em que vivemos demanda que possam ser ampliados os horizontes e, com isso, refletir sobre esses novos recursos de atendimento, tendo em vista uma elasticidade da técnica, em outras palavras, para além do interior dos consultórios.

Um estudo recente levantou dados a partir das impressões de psicólogos sobre seus atendimentos realizados on-line. Nesta pesquisa, ficou evidente que o tema precisa ser mais estudado e que existem diferenças nas modalidades de atendimento realizado no consultório e virtual. Ainda assim, é consenso entre os participantes que 0 atendimento on-line cresce e faz-se cada vez mais presente como mais uma forma de prestação de serviço do psicólogo (Magalhães, Bazoni \& Pereira 2019).

Ainda no trabalho citado, a relação de vínculo foi abordada e foi apontado que os pacientes atendidos on-line se envolvem no processo da mesma maneira que os atendidos de maneira presencial. Com base nisso, a pesquisa ressaltou que o sucesso de um processo terapêutico não está ligado ao aparelho tecnológico que media os atendimentos, mas, sim, à habilidade do psicólogo e à implicação e motivação do paciente no seu tratamento (Magalhães, Bazoni \& Pereira, 2019).

\section{Síncrono e assíncrono}

Os atendimentos psicológicos on-line podem ser realizados de duas maneiras. A primeira, no modelo síncrono, ou seja, uma comunicação imediata entre o psicólogo e o paciente, por meio de chats, áudios ou vídeos. Sendo assim, mediado pelas TICs, esse modelo tem uma interação instantânea, 0 que demanda um período de tempo contínuo entre as duas partes ao mesmo tempo (Siegmund, Jazen, Gomes \& Gauer, 2015).

Segundo Suler (2004), o modelo síncrono proporciona uma comunicação simultânea, auxiliando na espontaneidade da experiência e aproximando 0 on-line do presencial. No meio on-line, podem ocorrer interrupções no diálogo por intermédio de uma falha de conexão, um congelamento de imagem ou uma falha no áudio. Se a troca for realizada por meio de mensagens, 0 
tempo de digitação diminui o ritmo de conversa. No entanto, os erros, falhas e exclusões podem ser atribuídos como informações dessa comunicação.

0 atendimento psicológico on-line síncrono apresenta questões que podem ser vantajosas, como: 0 agendamento das sessões por um tempo definido e limitado, permitindo uma relação de compromisso entre psicólogo e paciente; a alternativa de um sentimento de presença ao estarem conectados simultaneamente; as pausas na conversa e a ausência na sessão são dados importantes para o processo terapêutico. Contudo, as desvantagens são: a dificuldade de agendar a sessão na existência de diferenças no fuso horário; um curto espaço de tempo para refletir e formular uma resposta; e a possibilidade do paciente vincular a terapia como uma consulta e não como um processo terapêutico (Suler, 2004).

A segunda maneira de atendimento psicológico on-line é a assíncrona. Nesta, a comunicação ocorre com um lapso de tempo, ou seja, o psicólogo e o paciente não estão simultaneamente conectados, o que não descarta a existência do vínculo. Por exemplo, na troca de e-mails, existe uma troca de informações, uma elaboração desse conteúdo e um retorno sobre o mesmo. Um ponto importante a ser destacado é que alguns pacientes preferem esse modelo de atendimento pela flexibilidade de tempo para ler e responder a esses e-mails (Siegmund et al., 2015).

De acordo com Suler (2004), o modelo assíncrono engloba a comunicação com um espaço de tempo entre uma conexão e outra, alongando, assim, a interação entre o psicólogo e o paciente, sem uma definição de limite de tempo. Todavia, a compreensão de compromisso pode ficar alterada ou confusa, pois as fronteiras de tempo ficam subjetivas e com a falsa sensação de poder responder a qualquer momento ou em qualquer lugar, mesmo que o paciente dedique um momento específico para ler ou escutar a mensagem.

Os ajustes necessários para estabelecer o compromisso terapêutico não anulam a possibilidade do paciente se conectar com o psicólogo em um espaço de tempo fluído que transcorre o processo terapêutico. Existem também sistemas de comunicação síncronos que podem ser utilizados de maneira assíncrona, como fóruns que podem permanecer por um longo período abertos para interações (Suler, 2004).

0 atendimento psicológico on-line assíncrono destaca temas que podemos considerar vantajosos, tais como: a flexibilidade para agendamento de atendimentos em fuso horário diferente, a versatilidade de responder quando tiver disponibilidade, a existência de uma área de reflexão elaborada do psicólogo e do paciente, além da questão sobre a impulsividade que pode ser trabalhada. No âmbito da desvantagem, temos pontos como: a dificuldade de estabelecer os limites profissionais de atendimento em relação a tempo e frequência, uma ausência de espontaneidade entre ambos e o sentimento de presença reduzido diante da não simultaneidade de encontros nos atendimentos (Suler, 2004).

\section{Considerações finais}

A partir das pesquisas realizadas, foi possível constatar que, apesar de 0 atendimento on-line ser regulamentado como uma prática possível de exercício da psicologia, poucos psicólogos aderiam e tinham conhecimento sobre essa modalidade antes da pandemia de Covid-19. Sendo assim, mesmo com o fato de os cadastros para atendimento on-line terem aumentado devido à orientação do Conselho Federal de Psicologia visando evitar atendimentos presenciais, ainda se faz necessário refletir sobre o tema para que se ampliem as possibilidades de atuação dos psicólogos.

Nesse sentido, com a globalização e o advento das tecnologias, como foi exposto no decorrer do texto, as pessoas passaram a interagir e comunicar-se por meio de novos formatos, fazendo-se, assim, necessário que os psicólogos também acompanhassem as mudanças e oferecessem outras possibilidades de escuta do sofrimento psíquico. Isso se dá principalmente diante do momento atual que vivemos, no qual antigas percepções precisaram ser reeditadas rapidamente e as relações têm sido atualizadas para o contexto on-line em vista da orientação de distanciamento físico.

Pôde-se também perceber que, apesar de serem formas distintas, os efeitos al cançados no atendimento on-line são muito similares à modalidade presencial, visto que existe a possibilidade de ser estabelecido um contrato e uma estruturação de setting, com base em uma escuta ética, que assegure a eficácia do processo seja por interação síncrona ou assíncrona. Para isso, faz-se necessário a constante atualização das normativas que regem a profissão e que validam a utilização das tecnologias de informação e comunicação.

\section{Referências}

Baldanza, R. F. (2006). Comunicação no ciberespaço: reflexões sobre a relação do corpo na interação e sociabilidade em espaço virtual. In XXIX Congresso Brasileiro de Ciências da Comunicação. Brasília, DF.

Barak, A., Hen, L., Boniel-Nissim, M. \& Shapira, N. A. (2008). A comprehensive review and a meta-analysis of the effectiveness of internet-based psychotherapeutic interventions. Journal of Technology in Human services, 26(2-4), 109-160. doi: 10.1080/15228830802094429

Barros, G. (2013). O Setting analítico na clínica cotidiana. Estudos de Psicanálise, (40), 71-78. Recuperado de http://pepsic.bvsalud.org/scielo.php?script=sci_arttext\&pid=S0100-34372013000200008\&lng=pt\&tlng=pt

Brizola, J.; Fantin, N. (2016) Revisão da Literatura e Revisão Sistemática da Literatura. Revista de Educação do Vale dos Arinos, 3(2). Recuperado de https://periodicos.unemat.br/index.php/relva/article/view/1738/1630.

Ceroni, M. L. V. (2017). Atendimento psicoterápico online e seus desdobramentos na relação mente e corpo. Revista Latino-americana de Psicologia Corporal, 4(6), 99-110. Recuperado de https://psicorporal.emnuvens.com. br/rlapc/article/view/55/95

Conselho Federal de Psicologia (2000). Resolução (FP no 03/2000. Regulamenta o atendimento psicoterapêutico mediado por computador. Brasília, DF. Recuperado de https://sites.usp.br/psicoterapiaonline/wp-content/ uploads/sites/543/2019/06/legislaca040_03_2000.pdf

Conselho Federal de Psicologia (2005a). Resolução (FP no 10/2005. Aprova o Código de Ética profissional do psicólogo. Brasília, DF. Recuperado de http://site.cfp.org.br/wp-content/uploads/2012/07/codigo-de-etica-psicologia.pdf

Conselho Federal de Psicologia (2005b). Resolução (FP no 12/2005. Regulamenta o atendimento psicoterapêutico e outros serviços psicológicos mediados por computador e revoga a Resolução (FP no 03/2000. Recuperado de https://sites.usp.br/psicoterapiaonline/wp-content/uploads/ sites/543/2019/06/Resolucao_CFP_012-2005.pdf

Conselho Federal de Psicologia (2012). Resolução (FP no 11/2012. Regulamenta os serviços psicológicos realizados por meios tecnológicos de comunicação a distância, o atendimento psicoterapêutico em caráter experimental e revoga a Resolução (FP no 12/2005. Recuperado de https://site.cfp.org. br/wp-content/uploads/2012/07/Resoluxo_CFP_nx_011-12.pdf

Conselho Federal de Psicologia (2018). Resolução (FP no 11/2018. Regulamenta a prestação de serviços psicológicos por meio de Tecnologias 
da Informação e Comunicação e revoga a Resolução (FP no 11/2012. Recuperado de https://site.cfp.org.br/wp-content/uploads/2018/05/RESOLU\%C3\%87\%C3\%830-N\%C2\%BA-11-DE-11-DE-MAI0-DE-2018.pdf Conselho Federal de Psicologia (2020a). Resolução (FP no 04/2020. Regulamenta serviços psicológicos prestados por meio de Tecnologias da Informação e Comunicação durante a pandemia do COVID-19. Recuperado de https://atosoficiais.com.br/cfp/resolucao-do-exercicio-profissional-n-4-2020-dispoe-sobre-regulamentacao-de-servicos-psicologicos-prestados-por-meio-de-tecnologia-da-informacao-e-da-comunicacao-durante-a-pandemia-do-covid-19

Conselho Regional de Psicologia do Rio Grande do Sul (2020b). Terapias online e por telefone são alternativas durante isolamento social. Recuperado de: http://www.crprs.org.br/noticias/terapias-online-e-por-telefone-sao-alternativas-durante-isolamento-social

Dockhorn, C. N. B. F. \& Macedo, M. (2008). A complexidade dos tempos atuais: reflexões psicanalíticas. Revista Argumento Psicologia, 54(26), 217-224. Recuperado de https://biblat.unam.mx/hevila/Psicologiaargumento/2008/vol26/n054/4.pdf

Elias, C., Silva, L., Martins, M., Ramos, N., Souza, M. \& Hipólito, R. (2012) Quando chega o fim?: uma revisão narrativa sobre terminalidade do período escolar para alunos deficientes mentais. SMAD. Revista eletrônica saúde mental álcool e drogas, 8(1), 48-53. Recuperado de http://pepsic.bvsalud.org/scielo.php?script=sci_arttext\&pi$d=$ S1806-69762012000100008\&lng=pt\&tlng=pt.

Ferreira, A. B. H. (1999). Novo Aurélio Século XXI: O dicionário da língua portuguesa. Rio de Janeiro: Editora Nova Fronteira.

Fink, B. (2017). Análise por telefone (variações na situação psicanalítica). In, B. Fink, Fundamentos da Técnica Psicanalítica: uma Abordagem Lacaniana para Praticantes (pp. 317-345) (C. Luchetta, B. A. Berger, trad.). São Paulo: Blucher; Karnac. (Obra original publicada em 2007).

Freud, S. (1912-1996). Recomendações aos médicos que exercem a psicanálise. In S. Freud, Edição Standard Brasileira das Obras Psicológicas Completas de Sigmund Freud (Vol. 12, pp. 123-133). Rio de Janeiro: Imago.

Freud, S. (1913-1996). Sobre o início do tratamento. In S. Freud, Edição Standard Brasileira das Obras Psicológicas Completas de Sigmund Freud (Vol. 12, pp. 137-161). Rio de Janeiro: Imago.

Galvão, C. L. (2016). Os sentidos do termo virtual em Pierre Lévy. Logeion: Filosofia da Informação, 3(1), 108-120. doi: 10.21728/logeion.2016v3n1. p108-120

Green, A. (2008). Orientações para uma psicanálise contemporânea (pp.5364). São Paulo: Imago.

Josephine, K., Josefine, L., Philipp, D., David, E. \& Harald, B. (2017). Internet-and mobile-based depression interventions for people with diagnosed depression: a systematic review and meta-analysis. Journal of Affective Disorders, 223, 28-40. Affective Disorders. 223. doi: 10.1016/j. jad.2017.07.02

Lévy, P. (1996). 0 que évirtual? (Neves, P. trad.). São Paulo: Editora 34.

Loucas, C. E., Fairburn, C. G., Whittington, C., Pennant, M. E., Stockton, S.\& Kendall, T. (2014). E-therapy in the treatment and prevention of eating disorders: A systematic review and meta-analysis. Behaviour Research and Therapy, 63, 122-131. doi: 10.1016/j.brat.2014.09.011.

Lucion, N. \& Knijnik, L. (2015). 0 Contrato. In: C. L. Eizirik, R. W. de Aguiar \& S. S. Schestatsky (Org.), Psicoterapia de Orientação Analítica: Fundamentos Teóricos e Clínicos (3. ed, pp.212-223). Porto Alegre: Artmed.

Magalhães, L. T., Bazoni, A. C. \& Pereira, F. N. (2019). Impressões de psicólogos clínicos acerca da orientação psicológica online. Revista Brasileira de Psicoterapia, 21(1), 53-69. doi: 10.5935/2318-0404.20190009

Neto, F. S. (2020). "O Brasil acima de tudo, Deus acima de todos": igreja, política e globalização. Vox Faifae: Revista de Teologia da Faculdade Fasseb, 10(1), 1-12. Recuperado de http://www.faifa.edu.br/revista/index.php/ voxfaifae/article/view/147/159
Nóbrega, S. B. (2015). Psicanálise on-line: finalmente saindo do armário?. Estudos de Psicanálise, (44), 145-150. Recuperado de http://pepsic. bvsalud.org/scielo.php?pid=S0100-34372015000200016\&script=sci_abstract\&tlng=en

Organização Pan-Americana de Saúde \& Organização Mundial da Saúde (2020). Folha Informativa - COVID-19 (Doença Causada Pelo Novo Coronavírus). Recuperado de https://www.paho.org/bra/index.php?option=com_content \&view $=$ article\&id $=6101:$ covid19\&ltemid $=875$

Pieta, M. A. M. (2014) Psicoterapia pela internet: A relação terapêutica (Tese de doutorado). Universidade Federal do Rio Grande do Sul, Porto Alegre, RS, Brasil. Recuperado de https://lume.ufrgs.br/handle/10183/144025

Proudfoot, J., Klein, B., Barak, A., Carlbring, P., Cuijpers, P., Lange, A., Ritterband, L. \& Andersson, G. (2011). Establishing guidelines for executing and reporting internet intervention research. Cognitive Behaviour Therapy, 40(2), 82-97. doi: 10.1080/16506073.2011.573807

Richards, D., Richardson, T., Timulak, L. \& McElvaney, J. (2015). The efficacy of internet-delivered treatment for generalized anxiety disorder: A systematic review and meta-analysis. Internet Interventions, 2(3), 272-282. doi: 10.1016/j.invent.2015.07.003

Rodrigues, C. G. \& Tavares, M. de A. (2016). Psicoterapia online: Demanda crescente e sugestões para regulamentação. Psicologia em Estudo, 21(4), 735-744. doi: 10.4025/psicolestud.v21i4.29658

Rosa, G. A. M. Santos, B. R. D. \& Faleiros, V. D. P. (2016). Opacidad de las fronteras entre real y virtual en la perspectiva de los usuarios del Facebook. Psicologia USP, 27(2), 263-272. doi: 10.1590/0103-656420130026

Rother, Edna Terezinha. (2007). Revisão sistemática X revisão narrativa. Acta Paulista de Enfermagem, 20 (2), v-vi. doi.org/10.1590/5010321002007000200001

Siegmund, G. Jazen, M. R., Gomes, W. B.\& Gauer, G. (2015). Aspectos éticos das intervenções psicológicas on-line no Brasil: Situação atual e desafios. Psicologia em Estudo, 20(3), 437-447. doi: 10.4025/psicolestud.v20i3.28478

Silva, J. A. M. da, Siegmund, G. \& Bredemeier, J. (2015). Crisis interventions in online psychological counseling. Trends in Psychiatry and Psychotherapy, 37(4), 171-182. doi: 10.1590/2237-6089-2014-0026

Simblett, S. Birch, J. Matcham, F., Yaguez, L. \& Morris, R. (2017). A systematic review and meta-analysis of e-mental health interventions to treat symptoms of posttraumatic stress. JMIR Mental Health, 4(2), e14. doi: 10.2196/mental.5558

Suler, J. R. (2004). Psychotherapy in cyberspace: A 5-dimensional model of online and computer-mediated psychotherapy. CyberPsychology and Behavior, 3(2), 151-159. doi: 10.1089/109493100315996

Witt, D.T.\& Rostirola, S. C. M. (2020). Conectivismo pedagógico: novas formas de ensinar e aprender no século XXI. Revista Thema, 16(4), 1012-1025. doi:10.15536/thema.V16.2019.1012-1025.1583 